\title{
Performance Analysis of Weld Image Classification Using Modified Resnet CNN Architecture
}

\author{
Mohanasundari $L^{a}$, Sivakumar $\mathbf{P}^{\mathrm{b}}$ and SenthilKumar $\mathbf{K} \mathbf{P}^{\mathrm{c}}$ \\ Assistant professor, Department of ECE, Saveetha Engineering College,Chennai, \\ India.

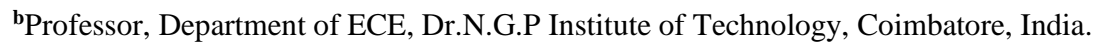 \\ ${ }^{\mathbf{c}}$ Assistant professor, Department of ECE, Easwari Engineering College, Chennai, \\ India.
}

Article History: Received: 11 January 2021; Accepted: 27 February 2021; Published online: 5 April 2021

\begin{abstract}
The detection and classifications of weld images is important for improving the quality of the joined materials during production period. In order to automate the classification of weld images in industry, this paper proposes an effective automatic method for the detection and classifications of the weld images into four different cases using deep learning methods. In this work, Convolutional Neural Network (CNN) is adopted for the weld image classification by modifying the internal architecture of the CNN architecture. This proposed ResNet CNN architecture is designed with three Convolutional layers, two numbers of pooling layers with activation layer and two numbers of Fully Connected Neural Networks (FCNN).The FCNN in proposed CNN architecture is designed with 15 internal hidden layers and each hidden layer is designed with 20 neurons which obtains high classification efficiency. The morphological activity functional methods are used on the classified weld images to detect the crack regions.
\end{abstract}

Keywords: Weld, CNN, Deep learning, FCNN, Classification accuracy

\section{Introduction}

The welding is the important process for manufacturing the different kind of materials. The quality of the material is based on the type of welding [3-5]. In general, though there are numerous types of welding available, four types of welds are frequently seen in many materials. They are Good weld case, Excess weld case, No weld case and Undercut weld case. In case of Good weld, the welding regions on the joined materials are very perfect and the weld is lasting for several years. Hence, this type of weld is known as perfect weld for all kind of materials. In case of Excess weld, the welding portion is exceeding on both joined materials. This type of weld is not perfect and not suitable for non-robust materials. In case of No-weld, the weld portion is not there on the joined materials. It is the worst case and hence it should be detected and eliminated during manufacturing process. In case of Undercut weld, the welding on the joined materials is not perfect. Fig.1 shows these four types of weld images used in industry process.

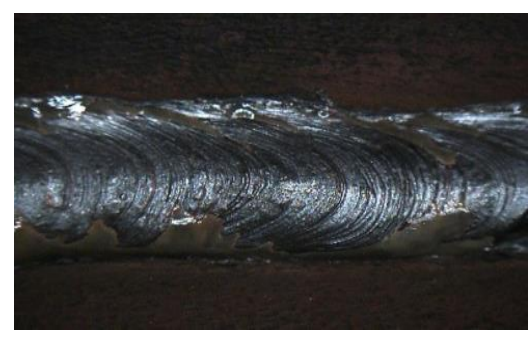

(a)

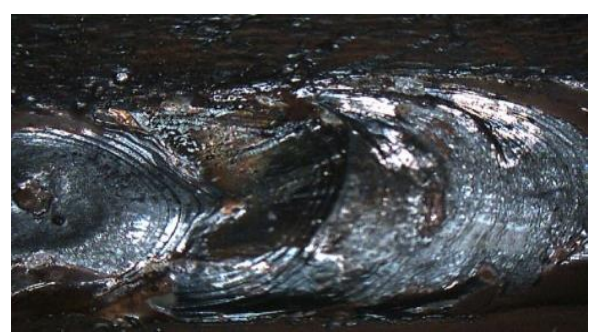

(b)

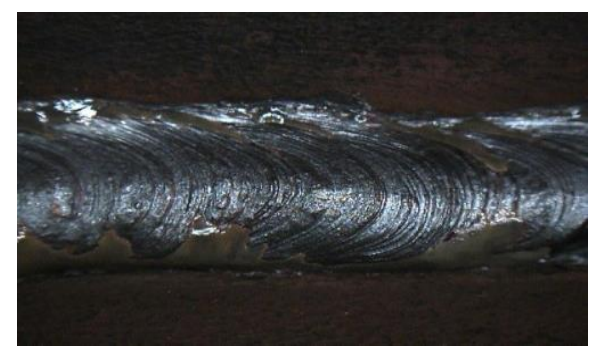

(b)

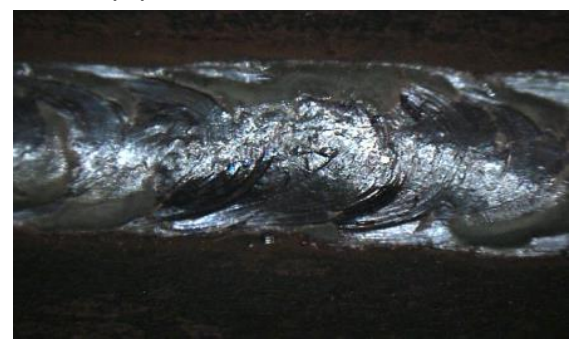

(d)

Figure 1 (a) Excess weld (b) Good weld (c) No weld (d) Under cut weld

Hence, the detection and classifications of weld images is important for improving the quality of the joined materials during production period. In order to automate the classification of weld images in industry, this paper proposes an effective automatic method for the detection and classifications of the weld images into four different cases using deep learning methods. In this work, $\mathrm{CNN}$ is adopted for the weld image classification by modifying the internal architecture of the $\mathrm{CNN}$ architecture. 
This paper is split into different sections. Section 2 states the conventional methods for weld image classifications in industry, section 3 states the proposed CNN architecture for weld image classifications, section 4 states the simulation results of the proposed $\mathrm{CNN}$ architecture and section 5 states the conclusion of the article.

\section{Literature survey}

Yang et al. (2020) computed multi level featuring parameters from the source weld images and these computed feature ser were analyzed and classified using unified deep learning neural networks. The authors used geometric and intensity based feature extraction process for training the deep learning architectures in this paper. The methods stated in this paper achieved $91.36 \%$ of classification accuracy on weld image dataset. ChirazAjmi et al. (2020) used deep learning method for detecting the cracked images in weld materials. The authors used AlexNet architecture for identifying the weld image types during the manufacturing process in industry. The authors used fine-tuning technique in the developed AlexNet CNN architecture for improving the classification accuracy of the detected weld images. Wu et al. (2019) used electromagnetic sensing diffusion method for finding the cracks on the welded images. The properties for weld portions were analyzed in this paper using various electro index parameters. The proposed methods in this article were applied on large number of weld images in open access dataset and these methods were validated by the manual process also. The error rate of this method was low as $1.56 \%$ and hence many industries were used this method for welding property analysis process. ArunMohana et al. (2018) analyzed different existing methods for crack detection process in welded images. The authors evaluated the performance of each existing methods in terms of number of index parameters. Many soft computing techniques which included deep learning architectures were discussed in this work. The welding quality was inspected by artificial neural network was concluded as one of the foremost methods for weld image process in industry. The methods were discussed on both live weld images and off line images also in order to validate the effectiveness of these existing methods.

Liu et al. (2017) used number of image processing algorithms for detecting the crack regions on the welded images. The eddy current thermal imaging method was used to detect and segment the cracked regions on the welded images. The authors used k-fold validation method to validate the simulation results of the crack region detection system. The authors applied their proposed eddy current thermal imaging method on large number of weld images and the authors obtained $90.6 \%$ of classification rate. Yang et al. (2015) used eddy current method for analyzing the detected cracked regions in welded images. The eddy properties of the welding portions of the welded images were trained by the linear classification approach and the trained results were used for the testing the linear classification approach in this paper. The authors applied their proposed eddy current based linear classifier on large number of weld images and the authors obtained $87.8 \%$ of classification rate.

\section{Proposed methodologies}

In this article, the weld images are classified into different classes using deep learning architecture. The conventional CNN deep learning architecture is modified and the weld images in four different cases are trained using this modified CNN architecture. Then, the same modified CNN architecture is used to classify the source weld image (which is to be tested) into four classes as Excess weld, Good weld, No weld and Undercut weld, respectively. The proposed flow of the weld image classification architecture in both training and testing category are depicted in Fig.2 (a) and Fig.2 (b), respectively.

The data augmentation process is used to increase the source weld image counts to improve the classification performance. This article uses time shifting function which includes both left and right shift, on the input source weld image to obtain high number of weld images in each weld cases.

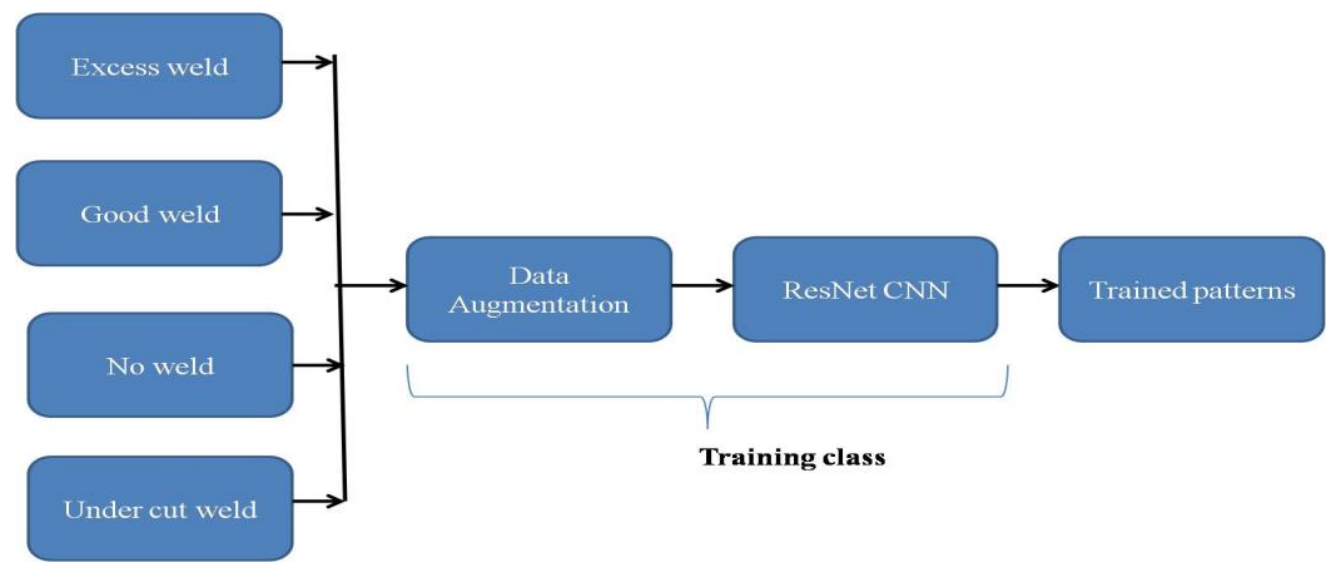

(a) 


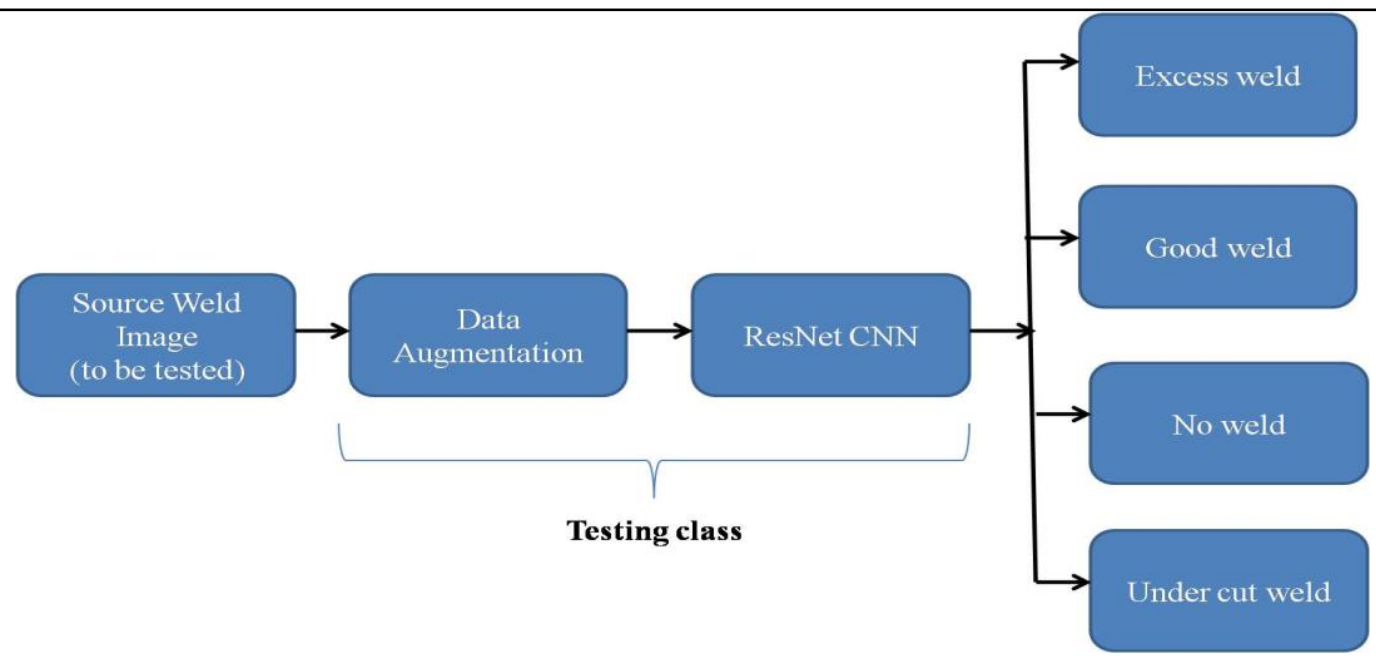

(b)

Figure 2 CNN classifications (a) Training class (b) Testing class

The existing ResNet CNN architecture is applied for weld image classifications, which classifies the weld image into four classes. This existing ResNet CNN architecture is designed with three Convolutional layers, two numbers of pooling layers with activation layer and unique FCNN [10-11]. The Convolutional layer 1 is designed with 32 numbers of Convolutional filters with a size of $7 * 7$ kernels. The Convolutional layer 2 is designed with 64 numbers of Convolutional filters with a size of $7 * 7$ kernels. The Convolutional layer 3 is designed with 128 numbers of Convolutional filters with a size of $7 * 7$ kernels. The source input weld image is multiplied with internal kernel of each Convolutional layer which produces the matrix. The size of this matrix is greater than the size of the source weld image size. Hence, this matrix is passed through the pooling layer in order to reduce the size of this matrix. The response of the pooling function may also contain negative value also. In order to eliminate such negative response, the response of pooling layer is passed through the activation layer. The response from the activation layer is passed through the FCNN which produces the final classification responses (four classes). The FCNN in existing CNN architecture is designed with 3 internal hidden layers and each hidden layer is designed with 15 neurons.

This proposed ResNet CNN architecture is designed with three Convolutional layers, two numbers of pooling layers with activation layer and two numbers of FCNN. The Convolutional layer 1 is designed with 128 numbers of Convolutional filters with a size of 5*5 kernels. The Convolutional layer 2 is designed with 256 numbers of Convolutional filters with a size of $5 * 5$ kernels. The Convolutional layer 3 is designed with 512 numbers of Convolutional filters with a size of $5 * 5$ kernels. The source input weld image is multiplied with internal kernel of each Convolutional layer which produces the matrix. The size of this matrix is greater than the size of the source weld image size. Hence, this matrix is passed through the Maximum pooling layer in order to reduce the size of this matrix. The average pooling layer in existing CNN architecture is replaced by the Average pooling function in the proposed CNN architecture. The response of the pooling function may also contain negative value also. In order to eliminate such negative response, the response of pooling layer is passed through the activation layer. The response from the activation layer is passed through the two numbers of FCNN which produces the final classification responses (four classes). The FCNN in proposed CNN architecture is designed with 15 internal hidden layers and each hidden layer is designed with 20 neurons which obtains high classification efficiency. The morphological activity functional methods(Sheelaet al. 2020) are used on the classified weld images to detect the crack regions.

Fig.3 (a) shows the Conventional ResNet CNN architecture and Fig.3 (b) shows the proposed ResNet CNN architecture.

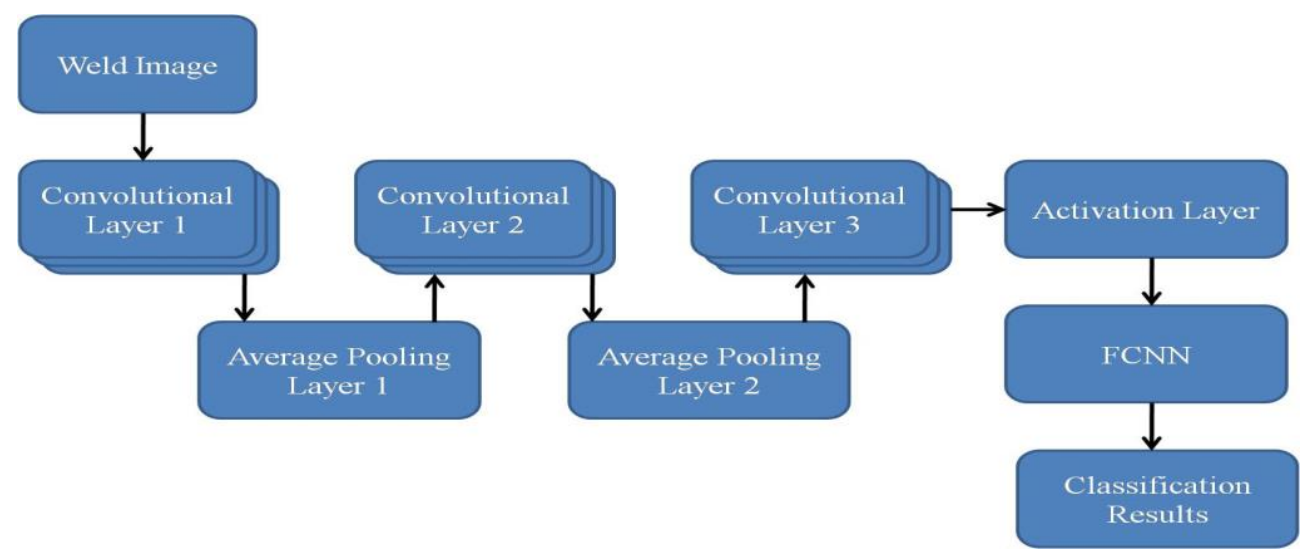


(a)

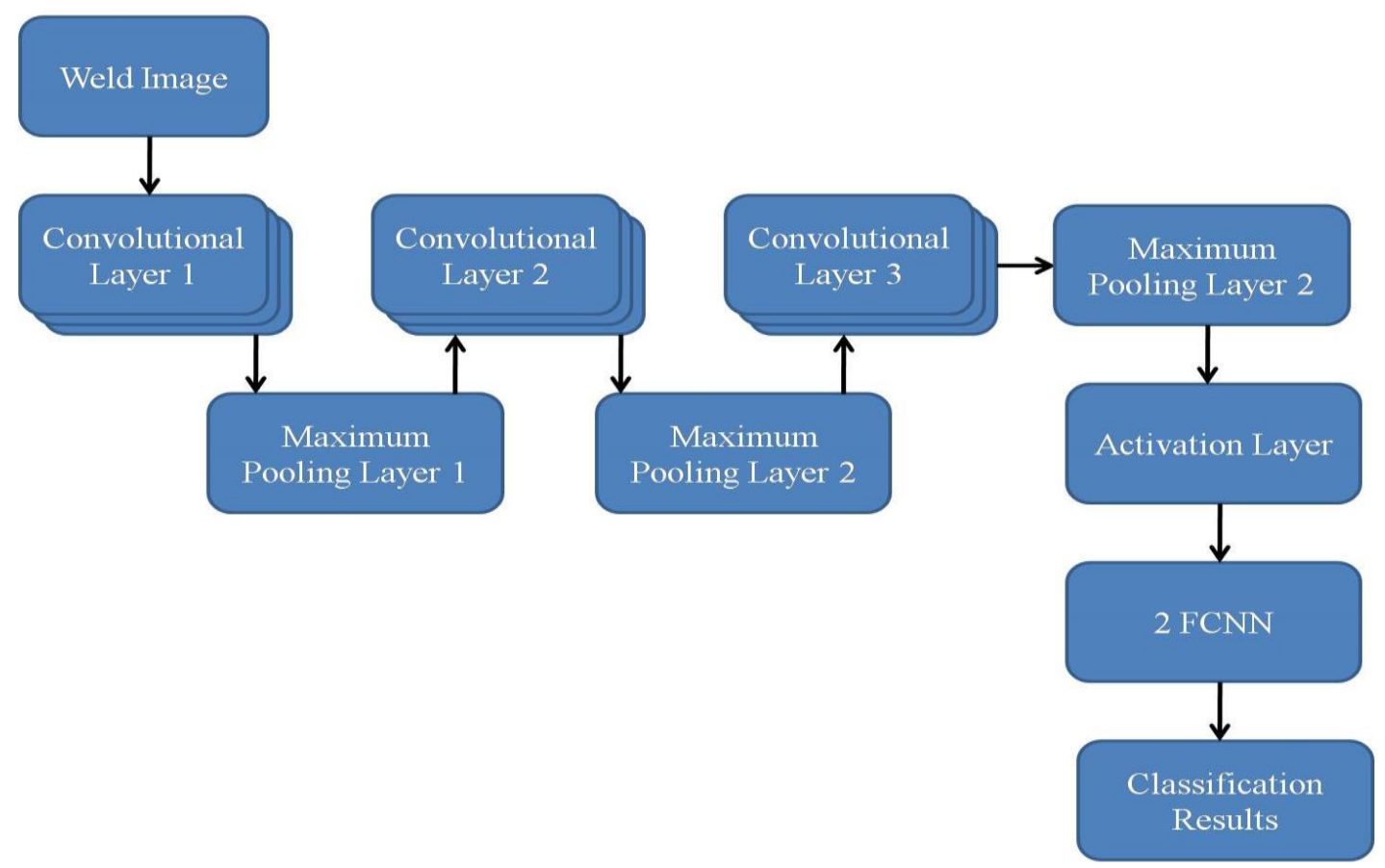

(b)

Figure 3 (a) Conventional ResNet CNN architecture [13,14] (b) Proposed ResNet CNN architecture

4. Results and Discussions

MATLAB R2018 is used in this article to simulate the proposed CNN architecture for automatic weld image classifications. Table 1 shows the analysis of performance of conventional and proposed ResNet CNN algorithm on weld image classifications. In this article, the number of weld images in Excess case is 55 and the number of weld images in Good case is 34, the number of weld images in No weld case is 78 and the number of weld images in undercut weld case is 75. Hence, the total number of weld images used in this article is about 242. The size of each weld image used in this article is about $128 * 128$ pixels as image width and height respectively. The conventional ResNet CNN architecture detects 50 number of excess weld case images and obtains $90.9 \%$ of CA, 32 number of Good case images and obtains $94.1 \%$ of CA, 76 number of No weld case images and obtains $97.4 \%$ of CA and 75 number of Undercut weld case images and obtains $94.6 \%$ of CA. Hence, the average CA of the weld image classification system using conventional ResNet CNN architecture is about $94.2 \%$.

The proposed ResNet CNN architecture detects 55 number of excess weld case images and obtains $100 \%$ of CA, 34 number of Good case images and obtains $100 \%$ of CA, 77 number of No weld case images and obtains $98 \%$ of CA and 75 number of Undercut weld case images and obtains $100 \%$ of CA. Hence, the average CA of the weld image classification system using conventional ResNet CNN architecture is about $99.5 \%$.

Table 1 Analysis of performance of conventional and proposed ResNetCNN algorithm on weld image classifications

\begin{tabular}{|c|c|c|c|c|c|}
\hline \multirow[t]{2}{*}{$\begin{array}{l}\text { Weld } \\
\text { case }\end{array}$} & \multirow[t]{2}{*}{$\begin{array}{c}\text { Numbe } \\
\text { r of images }\end{array}$} & \multicolumn{2}{|c|}{$\begin{array}{l}\text { Number of correctly classified } \\
\text { weld images }\end{array}$} & \multicolumn{2}{|c|}{$\begin{array}{l}\text { Classification accuracy (CA) } \\
(\%)\end{array}$} \\
\hline & & $\begin{array}{c}\text { Conventiona } \\
\text { I ResNet CNN } \\
\text { architecture }\end{array}$ & $\begin{array}{c}\text { Proposed } \\
\text { ResNet CNN } \\
\text { e }\end{array}$ & $\begin{array}{c}\text { Conventiona } \\
\text { I ResNet CNN } \\
\text { architecture }\end{array}$ & $\begin{array}{c}\begin{array}{c}\text { Proposed } \\
\text { ResNet CNN }\end{array} \\
\text { architectur } \\
\text { e }\end{array}$ \\
\hline $\begin{array}{l}\text { Excess } \\
\text { weld }\end{array}$ & 55 & 50 & 55 & 90.9 & 100 \\
\hline $\begin{array}{l}\text { Good } \\
\text { weld }\end{array}$ & 34 & 32 & 34 & 94.1 & 100 \\
\hline No weld & 78 & 76 & 77 & 97.4 & 98 \\
\hline $\begin{array}{l}\text { Undercu } \\
\mathrm{t} \text { weld }\end{array}$ & 75 & 71 & 75 & 94.6 & 100 \\
\hline & 242 & 229 & 241 & 94.2 & 99.5 \\
\hline
\end{tabular}


Further, the performance of the weld image classification system is analyzed using the following factors.

$$
\begin{aligned}
& \text { Sensitivity }(\mathrm{Se})=\frac{T r_{-} \mathrm{Po}}{T r_{-} \mathrm{Po}+\mathrm{Fa} a_{-} \mathrm{Ne}} \\
& \text { Specificity }(\mathrm{Sp})=\frac{\mathrm{Tr} r_{-} \mathrm{Ne}}{T r_{-} \mathrm{Ne}+\mathrm{Fa} \mathrm{F}_{-} \mathrm{Po}}
\end{aligned}
$$

Segmentation Accuracy $(S A)=\frac{T r_{-} P o+T r_{-} N e}{T r_{-} P o+F a_{-} N e+T r_{-} N e+F a_{-} P o}$

Where, $T r_{-} P o, T r_{-} N e$ are the detected crack pixels accurately and $F a_{-} P o, F a_{-} N e$ are the in accurately detected crack pixels.

Table 2 shows the analysis of weld image classification system using conventional and proposed ResNet CNN algorithm.

Table 2Analysis of weld image classification system using conventional and proposed ResNet CNN

\begin{tabular}{|c|c|c|c|c|c|c|}
\hline \multirow[t]{2}{*}{$\begin{array}{c}\text { Image } \\
\text { Sequences }\end{array}$} & \multicolumn{3}{|c|}{$\begin{array}{l}\text { Conventional ResNet CNN } \\
\text { architecture }\end{array}$} & \multicolumn{3}{|c|}{$\begin{array}{l}\text { Proposed ResNet CNN } \\
\text { architecture }\end{array}$} \\
\hline & $\begin{array}{l}\text { Se } \\
(\%)\end{array}$ & $\begin{array}{l}\text { Sp } \\
(\%)\end{array}$ & $\begin{array}{l}\text { SA } \\
(\%)\end{array}$ & $\begin{array}{l}\mathrm{Se} \\
(\%)\end{array}$ & $\begin{array}{l}\text { Sp } \\
(\%)\end{array}$ & $\begin{array}{l}\text { SA } \\
(\%)\end{array}$ \\
\hline 1 & 96.1 & 94.2 & 96.7 & 97.2 & 98.5 & 99.1 \\
\hline 2 & 96.7 & 95.1 & 97.1 & 98.1 & 98.9 & 98.9 \\
\hline 3 & 97.1 & 95.2 & 97.2 & 97.7 & 98.1 & 99.5 \\
\hline 4 & 97.1 & 94.1 & 98.5 & 97.5 & 98.7 & 99.7 \\
\hline 5 & 97.2 & 94.3 & 97.6 & 98.1 & 98.6 & 99.6 \\
\hline 6 & 97 & 94.5 & 97.1 & 97.6 & 98.5 & 98.5 \\
\hline 7 & 97.1 & 94.1 & 97.1 & 97.1 & 98.7 & 99.8 \\
\hline 8 & 95.6 & 97.9 & 98.1 & 98.6 & 98.1 & 99.9 \\
\hline 9 & 94.1 & 98.6 & 97.2 & 97.6 & 99.6 & 98.6 \\
\hline 10 & 92.1 & 97.1 & 97.1 & 97.8 & 98.5 & 99.7 \\
\hline Average & 96.01 & 95.51 & 97.37 & 97.73 & 98.62 & 99.33 \\
\hline
\end{tabular}
algorithm

Table 3 is the comparisons of CNN architectures on weld image classification system and Table 4 is the performance analysis of proposed crack detection method.

Table 3Comparisons of CNN architectures on weld image classification system

\begin{tabular}{|l|l|l|}
\hline \multirow{2}{*}{ Metrics } & $\begin{array}{l}\text { Conventional ResNet CNN } \\
\text { architecture }\end{array}$ & $\begin{array}{l}\text { Proposed ResNet CNN } \\
\text { architecture }\end{array}$ \\
\cline { 2 - 3 } & Experimental Results (\%) & Experimental Results (\%) \\
\hline Sensitivity & 96.01 & $\mathbf{9 7 . 7 3}$ \\
\hline Specificity & 95.51 & $\mathbf{9 8 . 6 2}$ \\
\hline Accuracy & 97.37 & $\mathbf{9 9 . 3 3}$ \\
\hline
\end{tabular}

Table 4 Performance analysis of proposed crack detection method

\begin{tabular}{|l|c|c|}
\hline & \multicolumn{2}{|c|}{ Experimental Results (\%) } \\
\hline Evaluation Parameters & $\begin{array}{c}\text { With data augmentation } \\
\text { process }\end{array}$ & $\begin{array}{c}\text { Without data augmentation } \\
\text { process }\end{array}$ \\
\hline Sensitivity & $\mathbf{9 7 . 7 3}$ & 92.12 \\
\hline
\end{tabular}


Performance Analysis of Weld Image Classification Using Modified Resnet CNN Architecture

\begin{tabular}{|l|c|c|}
\hline \hline Specificity & $\mathbf{9 8 . 6 2}$ & 93.78 \\
\hline Accuracy & $\mathbf{9 9 . 3 3}$ & 94.14 \\
\hline
\end{tabular}

Table 5 is the comparisons of proposed crack detection methods with conventional methods.

Table 5 Comparisons of proposed crack detection methods with conventional methods

\begin{tabular}{|l|l|l|l|c|}
\hline Methodology & $\begin{array}{l}\text { Se } \\
(\%)\end{array}$ & $\begin{array}{l}\text { Sp } \\
(\%)\end{array}$ & $\begin{array}{l}\text { SA } \\
(\%)\end{array}$ \\
\hline $\begin{array}{l}\text { Proposed work } \\
\text { (In this paper) }\end{array}$ & $\mathbf{9 7 . 7 3}$ & $\mathbf{9 8 . 6 2})$ & $\mathbf{9 9 . 5}$ \\
\hline Yang et al. (2020) & 95 & 95 & 96.33 & 92 \\
\hline $\begin{array}{l}\text { ChirazAjmi et al. } \\
(2020)\end{array}$ & 94 & 94 & 95 & 91 \\
\hline
\end{tabular}

\section{Conclusions}

This paper develops an automatic weld image classification system using deep learning architecture which modifies the existing CNN architecture in order to achieve high performance. The proposed ResNet CNN architecture obtains $97.73 \%$ of Se, $98.62 \%$ of Sp, $99.33 \%$ of SA and $99.5 \%$ of CA in this article. The proposed ResNet CNN architecture detects 55 number of excess weld case images and obtains $100 \%$ of CA, 34 number of Good case images and obtains $100 \%$ of CA, 77 number of No weld case images and obtains $98 \%$ of CA and 75 number of Undercut weld case images and obtains $100 \%$ of CA. Hence, the average CA of the weld image classification system using conventional ResNet CNN architecture is about 99.5\%. The simulation results are compared with existing methods.

\section{References}

1. ArunMohana and SumathiPoobal, "Crack detection using image processing: A critical review and analysis”, Alexandria Engineering Journal, Volume 57, Issue 2, June 2018, Pages 787-798.

2. Y. Wu et al., "Weld Crack Detection Based on Region Electromagnetic Sensing Thermography," in IEEE Sensors Journal, vol. 19, no. 2, pp. 751-762, 15 Jan.15, 2019.

3. J. Zhao B. Gao W. L. Woo F. Qiu G. Y. Tian "Crack evaluation based on novel circle-ferrite induction thermography" IEEE Sensors J. vol. 17 no. 17 pp. 5637-5645 Sep. 2017.

4. N. M. Bhattad S. S. Patil "System and method for identifying defects in welds by processing X-ray images" J. Brazilian Soc. Mech. Sci. Eng. pp. 1-8 2015.

5. R. Yang Y. He B. Gao G. Y. Tian J. Peng "Lateral heat conduction based eddy current thermography for detection of parallel cracks and rail tread oblique cracks" Measurement vol. 66 pp. 54-61 Apr. 2015.

6. Y. He G. Y. Tian M. Pan D. Chen H. Zhang "An investigation into eddy current pulsed thermography for detection of corrosion blister" Corrosion Sci. vol. 78 pp. 1-6 Jan. 2014.

7. Z. Liu G. Lu X. Liu X. Jiang G. Lodewijks "Image processing algorithms for crack detection in welded structures via pulsed eddy current thermal imaging" IEEE Instrum. Meas. Mag. vol. 20 no. 4 pp. 34-44 Aug. 2017.

8. Yang, L., Jiang, H. Weld defect classification in radiographic images using unified deep neural network with multi-level features. J IntellManuf (2020).

9. ChirazAjmi, Juan Zapata, Sabra Elferchichi,’Deep Learning Technology for Weld Defects Classification Based on Transfer Learning and Activation Features",Advances in Materials Science and Engineering, Volume 2020, Article ID 1574350, 2020, pp.1-14.

10. Feng, S., Zhou, H., \& Dong, H. (2019). Using deep neural network with small dataset to predict material defects. Materials and Design,162, 300-310.

11. Hou, W., Wei, Y., Guo, J., \& Jin, Y. (2018). Automatic detection of welding defects using deep neural network. Journal of Physics Conference Series,933, 012006.

12. Jiang, H., Wang, R., Gao, Z., Gao, J., \& Wang, H. (2019). Classification of weld defects based on the analytical hierarchy process and Dempster-Shafer evidence theory. Journal of Intelligent Manufacturing,30(4), 2013-2024.

13. Ahuja, S., Panigrahi, B.K., Dey, N. et al. Deep transfer learning-based automated detection of COVID-19 from lung CT scan slices. ApplIntell 51, 571-585 (2021).

14. Khan, R.U., Zhang, X. \& Kumar, R. Analysis of ResNet and GoogleNet models for malware detection. J ComputVirol Hack Tech 15, 29-37 (2019). 
Mohanasundari $L^{a}$, Sivakumar $P^{b}$ and SenthilKumar K $P^{c}$

15. Sheela, C.J.J., Suganthi, G. Morphological edge detection and brain tumor segmentation in Magnetic Resonance (MR) images based on region growing and performance evaluation of modified Fuzzy C-Means (FCM) algorithm. Multimed Tools Appl 79, 17483-17496 (2020). 